\title{
Search for hidden-photon dark matter with the FUNK experiment
}

D. Veberič ${ }^{a}$, A. Andrianavalomahefa ${ }^{a}$, K. Daumiller $^{a}$, B. Döbrich ${ }^{b}$, R. Engel ${ }^{* a}$, J. Jaeckel ${ }^{c}$, M. Kowalskid ${ }^{d}$, A. Lindner ${ }^{d}$, H.-J. Mathes ${ }^{a}$, J. Redondo ${ }^{f}$, M. Roth $^{a}$, T. Schwetz-Mangold ${ }^{a}$, C.M. Schäfer ${ }^{a}$, R. Ulrich ${ }^{a}$ [The FUNK Experiment]

${ }^{a}$ Institute for Nuclear Physics, Karlsruhe Institute of Technology (KIT), Karlsruhe, Germany

${ }^{b}$ Physics Department, CERN, Geneva, Switzerland

${ }^{c}$ Institute for Theoretical Physics, Heidelberg University, Germany

${ }^{d}$ Deutsches Elektronen Synchrotron (DESY), Zeuthen, Germany

${ }^{e}$ Department of Physics, Humboldt University, Berlin, Germany

${ }^{f}$ Department of Theoretical Physics, University of Zaragoza, Spain

*E-mail: ralph.engel@kit.edu

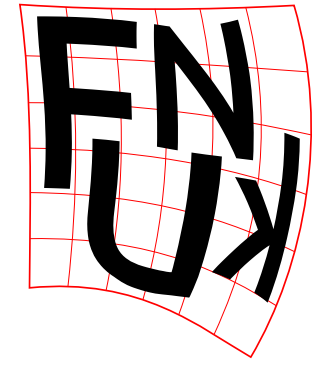

Many extensions of the Standard Model of particle physics predict a parallel sector of a new U(1) symmetry, giving rise to hidden photons. These hidden photons are candidate particles for cold dark matter. They are expected to kinetically mix with regular photons, which leads to a tiny oscillating electric-field component accompanying dark matter particles. A conducting surface can convert such dark matter particles into photons which are emitted almost perpendicularly to the surface. The corresponding photon frequency follows from the mass of the hidden photons. In this contribution we present a preliminary result on a hidden photon search in the visible and near-UV wavelength range that was done with a large, $14 \mathrm{~m}^{2}$ spherical metallic mirror and discuss future dark matter searches in the $\mathrm{eV}$ and sub-eV range by application of different detectors for electromagnetic radiation.

35th International Cosmic Ray Conference - ICRC2017

10-20 July, 2017

Bexco, Busan, Korea

${ }^{*}$ Speaker. 

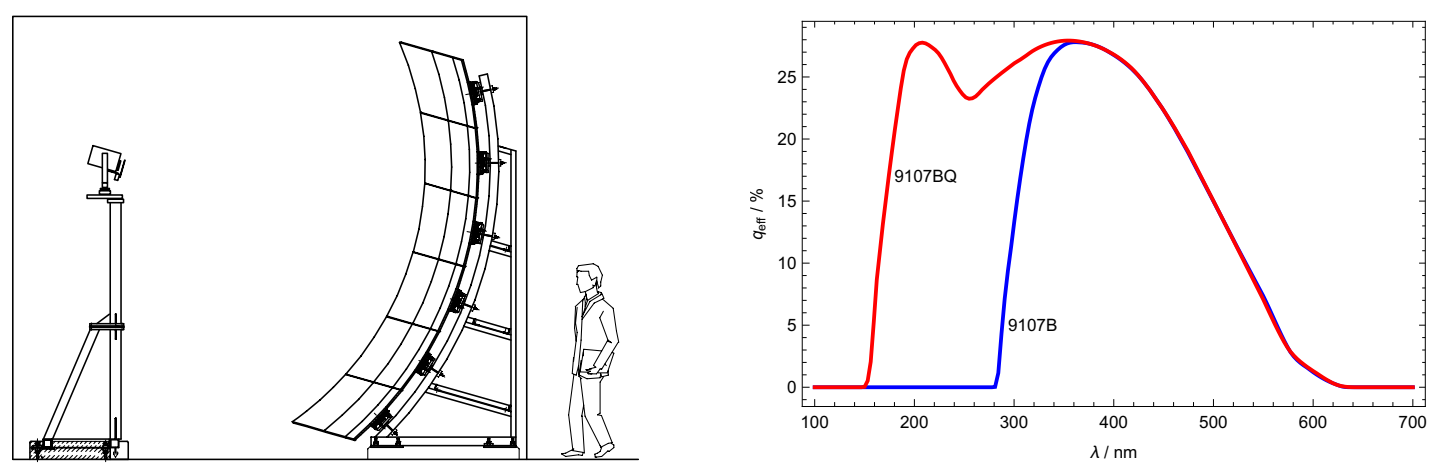

Figure 1: Left: Schematic view of the FUNK experiment with a pillar supporting the PMT camera in the center of the spherical mirror consisting of $6 \times 6$ segments. The box outlines a $495 \times 431 \times 435 \mathrm{~cm}^{3}$ experimental volume. Right: The quantum efficiency of the PMT used as photon sensor [19]. The UVextended sensitivity of the used PMT 9107BQ is shown in red.

\section{Introduction}

There is convincing astrophysical and cosmological evidence that a large fraction of the energy density in the universe must be composed of invisible non-baryonic matter or dark matter (DM) [1]. The most explored options for explaining DM are extensions of the Standard Model (SM) of particle physics predicting weakly-interacting massive particles (WIMPs). In recent years attention has been turned also to alternative scenarios involving weakly-interacting slim particles (WISPs) such as axions, axion-like particles, or hidden photons (HP) [2]. WISPs could be non-thermally produced in the early universe and survive as cold DM until today (see e.g. [3, 4, 5, 6] for reviews).

HP are additional light U(1) gauge bosons that kinetically mix with the SM photons [7]. The effective Lagrangian can be written as

$$
\mathscr{L}=-\frac{1}{4}\left(F_{\mu v} F^{\mu v}+X_{\mu v} X^{\mu v}\right)+J^{\mu} A_{\mu}+\frac{m^{2}}{2} X_{\mu} X^{\mu}-\frac{\chi}{2} F_{\mu v} X^{\mu v},
$$

where $F_{\mu \nu}=\partial_{\mu} A_{v}-\partial_{v} A_{\mu}$ is the field strength tensor of the photon and $X_{\mu \nu}$ that of the corresponding HP field, and $J^{\mu}$ the charge current.

Large phase space regions of the HP mass $m$ and mixing parameter $\chi$ are compatible with the observed DM signatures [8, 9]. Many different methods are applied to search for hidden photons or to derive constraints on their parameters. For example, laboratory experiments include haloscopes, helioscopes, and light-shining-through-a-wall methods [8].

In this paper we report about a search for HP as dominant DM particles with FUNK (Finding $\mathrm{U}(1) \mathrm{s}$ of a Novel Kind). FUNK is an experiment that is based on the recently proposed dish-antenna method $[10,11]$. A similar experiment, although with smaller sensitivity, has been performed in Tokyo $[12,13]$.

\section{The FUNK experiment}

In FUNK, a spherical mirror is used for searching for HP [10, 11]. When HP cross the metallic surface of the mirror, faint electromagnetic waves are emitted almost perpendicularly to the conducting surface. In the case of a spherical mirror these photons are focused on the radius point of 

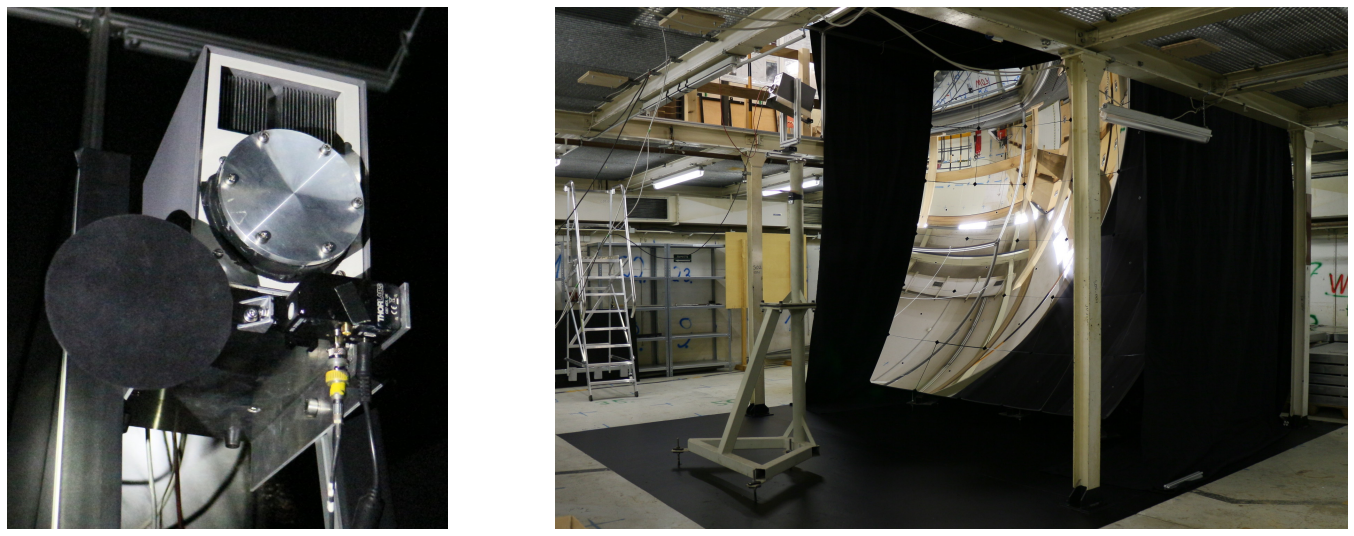

Figure 2: Left: A photograph of the PMT camera (closed with circular aluminum lid) with a Thorlabs motorized flipper carrying an obscuring shutter (black circle in front, in the "open" position). Right: The photograph of the FUNK experimental area, which is wrapped on top and the sides with a double-layer cover (black plastic sheet over a cotton curtain). The ground is treated with a mat-black paint.
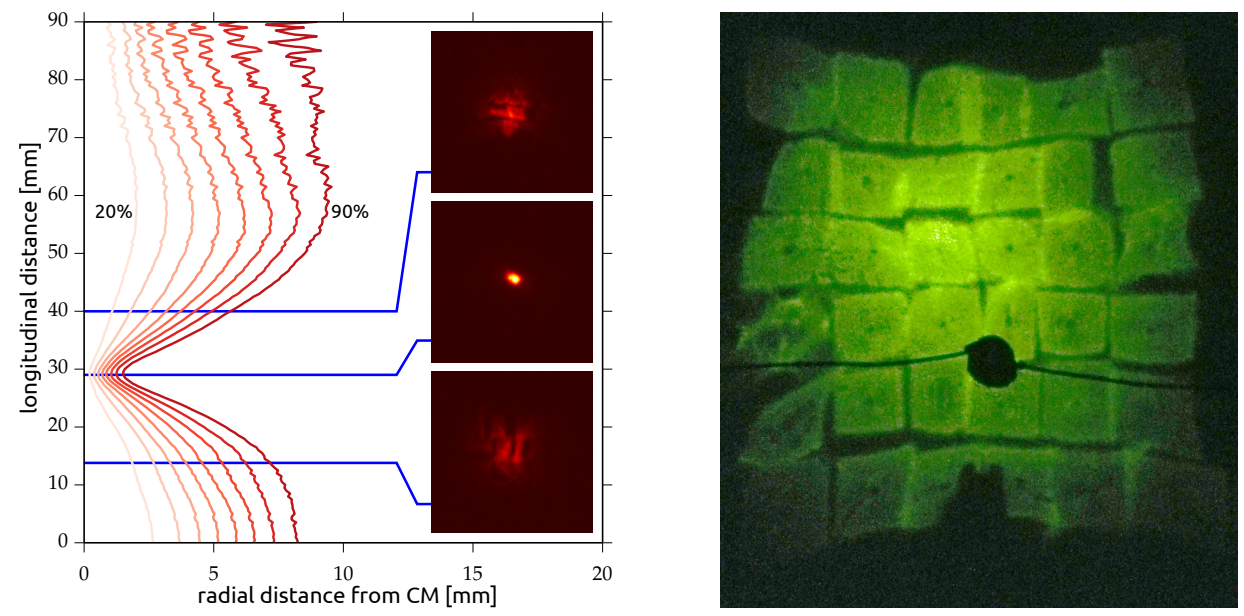

Figure 3: Left: Contours of radial distance from the image center of mass (CM) containing certain amount of total light (from 20\% to 90\% in steps of 10\%) for the final, realigned assembly of the mirror. The three insets show spot images in various positions marked by blue lines (middle line for the smallest spread). $90 \%$ of the light is contained within a spot of radius $\lesssim 2 \mathrm{~mm}$. Right: An image obtained when the LED is driven off-center towards the mirror. Reflected beams are not converged yet so that they form a matrix where each of the green squares corresponds to individual mirror segments, revealing their aberrations. The central black dot is a shadow of the LED with two horizontal power cables.

the mirror. Placing various detectors in the radius point allows us to search for a photon signal in different wavelength ranges, corresponding to different HP masses. The DM nature of a possible signal can be verified by observation of the expected small daily and seasonal movements of the spot $[11,14,15]$.

Mirror. For the purposes of the FUNK experiment we reused prototype mirror segments that were originally produced for the Schmidt telescopes of the fluorescence detector [16] of the Pierre Auger Observatory [17]. At KIT, we assembled them inside of a windowless air-conditioned experimental 

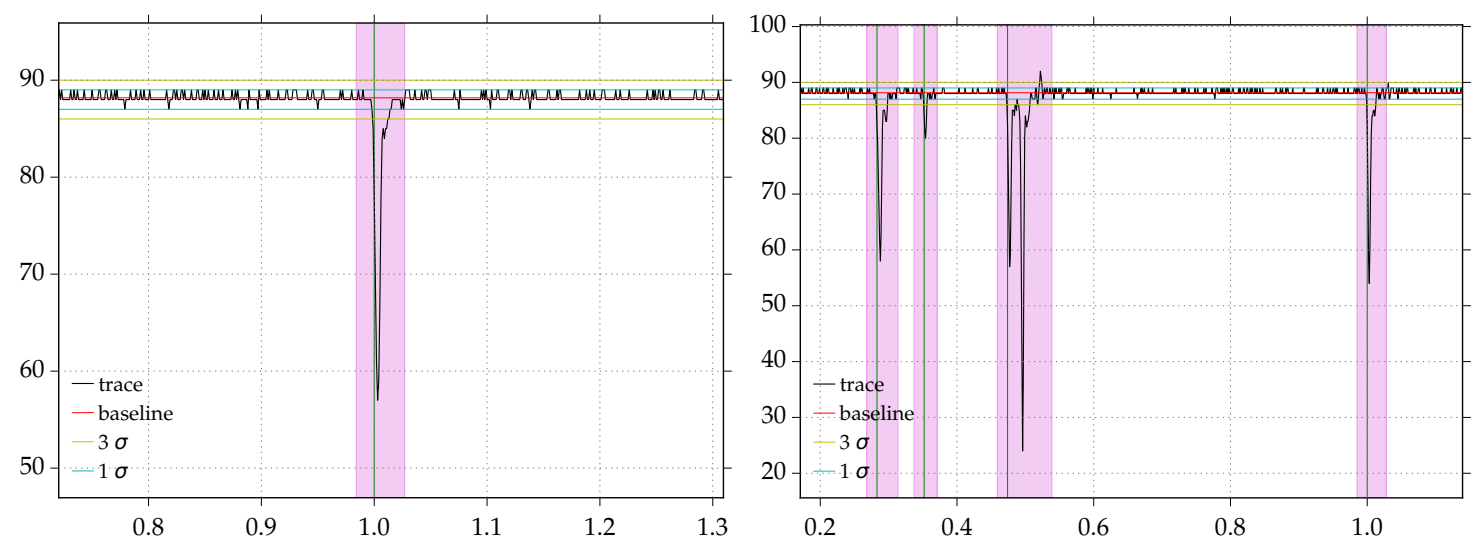

Figure 4: Left: An example of a typical single-photon trace. Right: An example of a trace with multiple pulses within the $1.6 \mu$ s trigger window which gets rejected by the cosmic-ray cut.
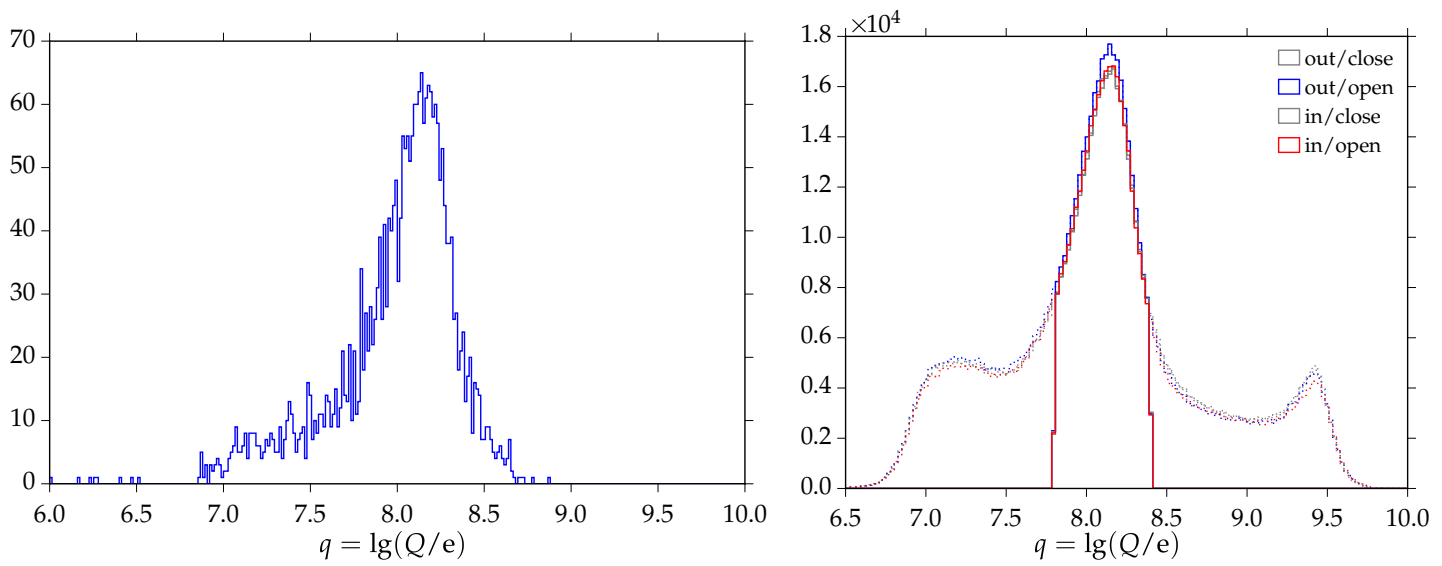

Figure 5: Left: Charge distribution for single photo-electron signals taken with an LED flasher; Right: Charge distribution of a FUNK run (without external light source).

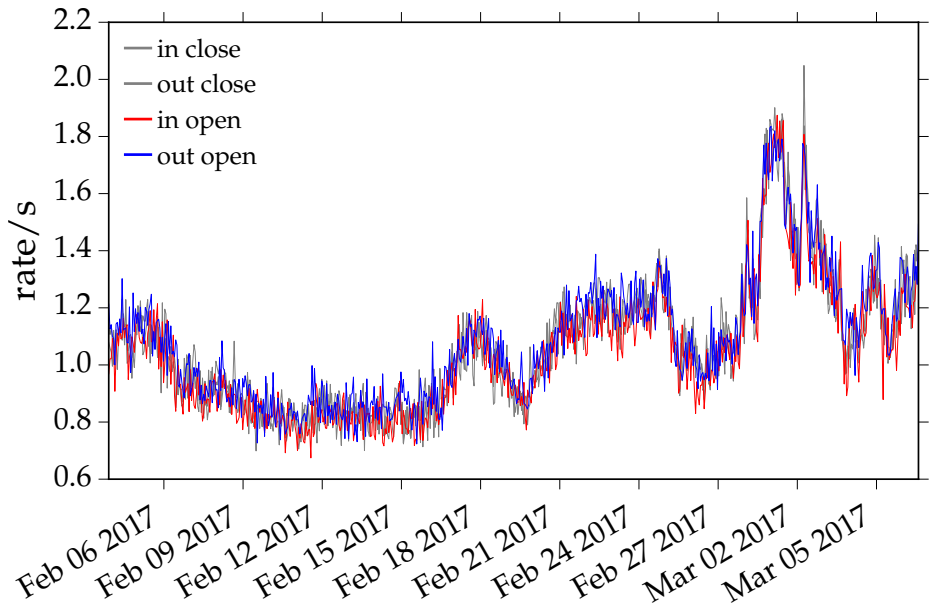

Figure 6: Raw count-rate for individual 60 s runs of the February 2017 measurement. 


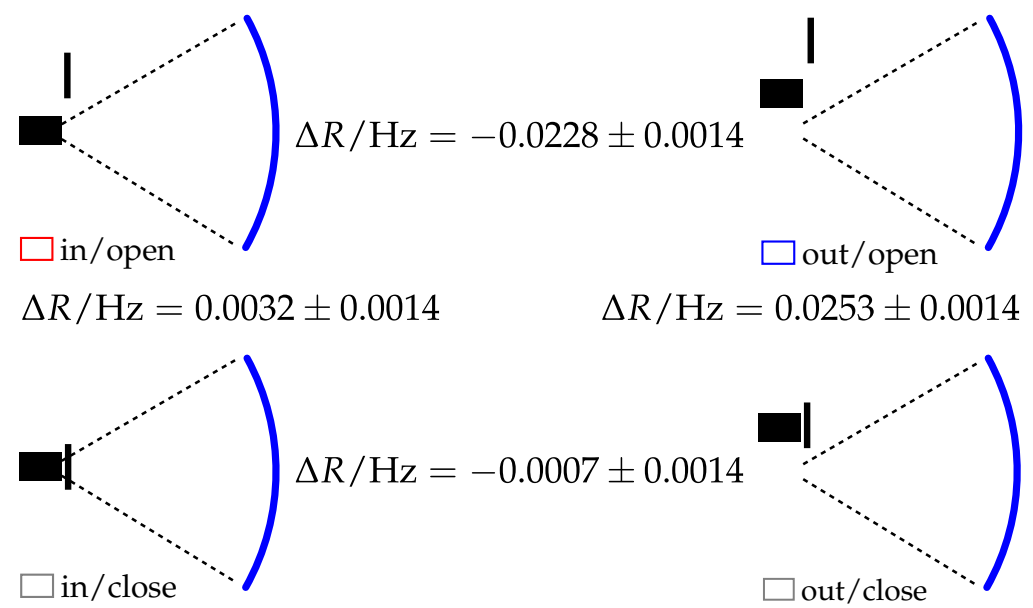

Figure 7: Differences in the counting rate with the PMT in in/out position with open/close shutter.

hall of $\sim 20 \mathrm{~m}$ diameter with $2 \mathrm{~m}$ thick concrete walls. The mirror represents a portion of the surface of a sphere with a radius of $3.4 \mathrm{~m}$ and total area of the mirror front surface is $14.56 \mathrm{~m}^{2}$ (see Fig. 1). For a more detailed description of the mirror we refer to the Ref. [18]. The whole experimental area with dimensions $495 \times 431 \times 435 \mathrm{~cm}^{3}$ has been additionally covered with a double-layer light shield (black plastic sheet over a cotton curtain) and the ground was treated with a non-reflective black paint, as can be seen in Fig. 2.

To quantify the optical quality of the mirror we built a movable imaging platform at the center of the mirror sphere. It consisted of a SMD LED as a light source, placed very close to the center of the sphere, which projected an image onto a frosted-glass screen. This image was then observed with a CCD camera (see the three insets in Fig. 3-left) and a movable linear stage enabled us to obtain a cross-section of the converging light beams from individual mirror segments. The mirror segments were adjusted individually to minimize the size of the image. The Fig. 3-left shows a scan through the central region after alignment, with contour lines in steps of $10 \%$ indicating the amount of light for various distances away from the central point. As can be seen in Fig. 3, the spot that contains $90 \%$ of all light has a radius of $\sim 2 \mathrm{~mm}$. Note that the seasonal oscillation of the HP spot due to the relative movement of the mirror in the DM frame is of the same order [11].

Photomultiplier. After the preparation of the mirror we performed a test measurement with a cooled PCO sensicam VGA CCD camera, the results of which have been presented in Ref. [18]. For the present measurement we chose a $29 \mathrm{~mm}$ diameter photomultiplier (PMT) ET 9107BQ [19] with a blue-green sensitive bialkali photocathode with the quantum efficiency extended into the ultra-violet range (see Fig. 1-right). The PMT has a high gain at relatively low voltages, peak quantum efficiency of around $28 \%$, and excellent single electron and pulse-height resolution, which makes it well-suited for photon counting applications. The active part of the photocathode has a diameter of $25 \mathrm{~mm}$ and is thus large enough to capture the spot of the potential DM signal even in the presence of seasonal movements.

The PMT was placed into a Fact-50 enclosure (see Fig. 2-left), which is capable of lowering the temperature of the PMT thermoelectrically by up to $50 \mathrm{~K}$, relatively to the ambient. Nevertheless, 
for the preliminary measurement presented in this proceeding, the cooling capability was not yet used, mostly because we wanted to avoid any unneccessary glass material in front of the PMT which could produce Cherenkov light by cosmic-ray muons passing through.

The PMT camera has been placed at the center of the spherical mirror (see Fig. 1-left) and equipped with an additional Thorlabs motorized flipper MFF101 carrying a shutter that can obscure the opening of the PMT to a very high degree. The operating high-voltage setting was chosen to be $1050 \mathrm{~V}$, close to a knee feature observed in a voltage scan of the PMT dark-current rate [20].

Data acquisition. Signals from the PMT were digitized with the PicoScope 6404D [21]. Examples of triggered traces can be seen in Fig. 4, where a nice, single-photon (SP) pulse can be observed on the left, while on the right we are showing an example of a trace that contained several pulses in the $3.2 \mu$ s trigger window. Such traces were discarded since they clearly belong to signals produced by cosmic-ray showers. Based on measurements of the SP charge spectrum with an LED flasher setup (Fig. 5-left), we also applied a cut on the allowed range of observed charges, as seen in Fig. 5-right. From the SP measurements we also estimated that the efficiency of such a cut on a SP signals is $75 \%$.

\section{Preliminary results}

The resulting counts for the February 2017 run can be seen in Fig. 6. The data was taken in cycles of $60 \mathrm{~s}$ in the two positions of the PMT camera, with the sensitive area "in" the center of the spherical mirror and $8 \mathrm{~cm}$ "out" of the center, and with the shutter "open" or "close" in both positions, thus resulting in four possible combinations, as schematically shown in Fig. 7. The average rate of the whole February run, after the quality selections, is $R=0.535 \mathrm{~Hz}$ and the differences between the rates in the four different configurations are shown in Fig. 7.

The difference of the count rates between "open" and "close" for the PMT being in the radius point is the measurement we are interested in. With the PMT window open there are $\Delta R=0.0032 \pm 0.0014 \mathrm{~Hz}$ more counts registered than with the PMT closed. If we ignore any possible sys. uncertainties, we would obtain the limit shown in Fig. 8 as "stat" for Funk. For determining possible sys. uncertainties that might be related to temperature changes or the limited accuracy of determining the measurement time, we also compare the rates with the covered PMT in and out of the radius point. The two count rates agree very well within the stat. uncertainty $(\Delta R=0.0007 \pm 0.0014 \mathrm{~Hz})$. Another cross check is the comparison of the count rates for the open PMT in and out of the radius point. We found a significant larger count rate if the PMT is open and outside of the radius point, which is possibly related to the different imaging properties of the setup in the two positions. Measurements are in progress to better understand this observation. For the time being we treat this difference of rates $(\Delta R \approx 0.025 \mathrm{~Hz})$ as an upper limit of the overall sys. uncertainty of our measurements and use this number to derive a preliminary upper limit [14] on the magnitude of the mixing parameter $\chi$ in the sensitivity range of the PMT, see the FUNK "syst" in Fig. 8.

\section{Conclusions and future plans}

We performed a search for HP dark matter with a metallic spherical mirror of $14 \mathrm{~m}^{2}$ in the visible 


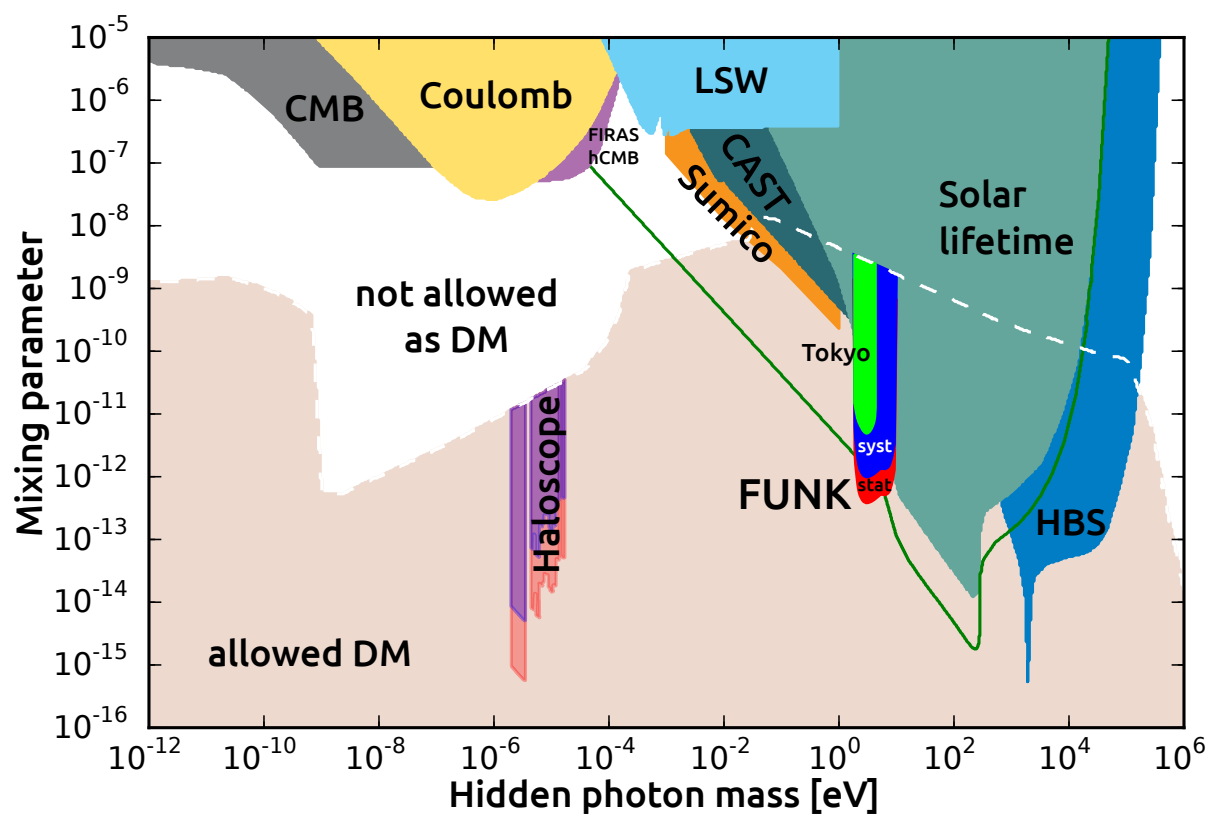

Figure 8: This Figure is an adapted and updated version of the figure in [3, 5], with the dark matter area from [8] and including new data and limits from [27, 28], in particular results from the Tokyo dish experiment $[12,13]$ (light green). The preliminary result for the FUNK exclusion area in the mixing parameter vs. hidden photon mass parameter space is shown in blue (for the systematics-bound result) and in red (for the statistics-bound result). Meaning of some other labels: measurements on cosmic microwave background (CMB), light-shining-through-wall experiments (LSW), horizontal-branch stars (HBS), and the green line corresponds to improved solar limits [24, 25]; for summary and labels see [26].

and near-UV wavelength range. No signal was found. Because the detailed analysis of the data is still ongoing we have reported here only preliminary results with a maximally conservative estimate of possible systematic uncertainties.

In the near future we are planning further measurements a fully cooled PMT, for which we expect reduced number of background counts. In addition we intend to extend the search for possible HP-DM into the MHz [22], GHz [23], and THz range.

Acknowledgments. We gratefully acknowledge partial support from the Helmholtz Alliance for Astroparticle physics (HAP), funded by the Initiative and Networking Fund of the Helmholtz Association.

\section{References}

[1] G.B. Gelmini, TASI 2014 lectures: The hunt for dark matter, NSF-KITP-15-005, [arXiv: 1502.01320].

[2] A.E. Nelson and J. Scholtz, Dark Light, Dark Matter and the Misalignment Mechanism, Phys. Rev. D 84 (2011) 103501, [arXiv: 1105.2812].

[3] J. Jaeckel and A. Ringwald, The Low-Energy Frontier of Particle Physics, Ann. Rev. Nucl. Part. Sci. 60 (2010) 405, [arXiv: 1002.0329].

[4] A. Ringwald, Searching for axions and ALPs from string theory, J. Phys. Conf. Ser. 485 (2014) 012013, [arXiv: 1209.2299]. 
[5] J. Jaeckel, A force beyond the Standard Model - Status of the quest for hidden photons, Frascati Phys. Ser. 56 (2012) 172, [arXiv: 1303.1821].

[6] K. Baker et al., The quest for axions and other new light particles, Annalen Phys. 525 (2013) A93-A99, [arXiv: 1306.2841].

[7] B. Holdom, Two U(1)'s and Epsilon Charge Shifts, Phys. Lett. B 166 (1986) 196.

[8] P. Arias, D. Cadamuro, M. Goodsell, J. Jaeckel, J. Redondo, and A. Ringwald, WISPy cold dark matter, JCAP 06 (2012) 013, [arXiv: 1201.5902].

[9] P.W. Graham, J. Mardon, and S. Rajendran, Vector Dark Matter from Inflationary Fluctuations, Phys. Rev. D 93 (2016) 103520, [arXiv: 1504.02102].

[10] D. Horns, J. Jaeckel, A. Lindner, A. Lobanov, J. Redondo, and A. Ringwald, Searching for WISPy Cold Dark Matter with a Dish Antenna, JCAP 04 (2013) 016, [arXiv: 1212.2970].

[11] J. Jaeckel and J. Redondo, An antenna for directional detection of WISPy dark matter, JCAP 11 (2013) 016.

[12] J. Suzuki, T. Horie, Y. Inoue, and M. Minowa, Experimental Search for Hidden Photon CDM in the eV mass range with a dish antenna, JCAP 09 (2015) 042, [arXiv: 1504.00118].

[13] J. Suzuki, Y. Inoue, T. Horie, and M. Minowa, Hidden photon CDM search at Tokyo, DESY-PROC-2015-02, [arXiv: 1509.00785].

[14] B. Döbrich, K. Daumiller, R. Engel, M. Kowalski, A. Lindner, J. Redondo, and M. Roth, Hidden Photon Dark Matter Search with a Large Metallic Mirror, Proc. 10th Patras Workshop on Axions, WIMPs and WISPS, CERN, 29 June - 4 July 2014, [arXiv: 1410.0200].

[15] J. Jaeckel, S. Knirck, Directional Resolution of Dish Antenna Experiments to Search for WISPy Dark Matter, JCAP 01 (2016) 005, [arXiv: 1509.00371].

[16] J. Abraham et al. [Pierre Auger Collaboration], The Fluorescence Detector of the Pierre Auger Observatory, Nucl. Instrum. Meth. A 620 (2010) 227-251, [arXiv: 0907.4282].

[17] A. Aab et al., The Pierre Auger Cosmic Ray Observatory, Nucl. Instrum. Meth. A 798 (2015) 172-213, [arXiv: 1502.01323].

[18] D. Veberič et al., Search for dark matter in the hidden-photon sector with a large spherical mirror, Proc. 34th Int. Cosmic Rays Conf. 2015, The Hague, The Netherlands, PoS (ICRC2015) 1191, [arXiv: 1509.02386].

[19] ET Enterprises, $9107 B$ series data sheet.

[20] B. Döbrich et al., The FUNK search for Hidden Photon Dark Matter in the eV range, Proc. of Int. Conference on the Structure and Interactions of the Photon (PHOTON 2015) and 21th International Workshop on Photon-Photon Collisions and Int. Workshop on High Energy Photon Linear Colliders, 15-19 Jun 2015, Novosibirsk, Russia, [arXiv: 1510.05869].

[21] Pico Technology, http://www.picotech.com.

[22] A. Aab et al. [Pierre Auger Collaboration], Probing the radio emission from air showers with polarization measurements, Phys. Rev. D 89 (2014) 052002.

[23] R. Šmída et al. [CROME experiment], First Experimental Characterization of Microwave Emission from Cosmic Ray Air Showers, Phys. Rev. Lett. 113 (2014) 221101.

[24] H. An, M. Pospelov, and J. Pradler, New stellar constraints on dark photons, Phys. Lett. B 725 (2013) 190, [arXiv: 1302.3884].

[25] J. Redondo and G. Raffelt, Solar constraints on hidden photons re-visited, JCAP 08 (2013) 034, [arXiv: 1305.2920].

[26] M. Goodsell, J. Jaeckel, J. Redondo, and A. Ringwald, Naturally light hidden photons in LARGE volume string compactifications, JHEP 11 (2009) 027.

[27] H. An, M. Pospelov, and J. Pradler, New stellar constraints on dark photons, Phys. Lett. B 725 (2013) 190-195, [arXiv: 1302.3884].

[28] N. Vinyoles, A. Serenelli, F. L. Villante, S. Basu, J. Redondo, and J. Isern, New axion and hidden photon constraints from a solar data global fit, JCAP 10 (2015) 015, [arXiv: 1501.01639]. 